\title{
The 2-10 keV luminosity as a Star Formation Rate indicator
}

\author{
P. Ranalli ${ }^{1}$, A. Comastri ${ }^{2}$, and G. Setti ${ }^{1}$ \\ 1 Dipartimento di Astronomia, Università di Bologna, via Ranzani 1, 40127 Bologna, Italy \\ 2 INAF - Osservatorio Astronomico di Bologna, via Ranzani 1, 40127 Bologna, Italy
}

Received 30 July 2002 / Accepted 4 November 2002

\begin{abstract}
Radio and far infrared luminosities of star-forming galaxies follow a tight linear relation. Making use of ASCA and BeppoSAX observations of a well-defined sample of nearby star-forming galaxies, we argue that tight linear relations hold between the X-ray, radio and far infrared luminosities. The effect of intrinsic absorption is investigated taking NGC 3256 as a test case. It is suggested that the hard X-ray emission is directly related to the Star Formation Rate. Star formation processes may also account for most of the $2-10 \mathrm{keV}$ emission from LLAGNs of lower X-ray luminosities (for the same FIR and radio luminosity). Deep Chandra observations of a sample of radio-selected star-forming galaxies in the Hubble Deep Field North show that the same relation holds also at high $(0.2 \lessgtr z \lessgtr 1.3)$ redshift. The X-ray/radio relations also allow a derivation of $\mathrm{X}$-ray number counts up to very faint fluxes from the radio $\log N-\log S$, which is consistent with current limits and models. Thus the contribution of star-forming galaxies to the X-ray background can be estimated.
\end{abstract}

Key words. X-rays: galaxies - radio continuum: galaxies - galaxies: high-redshift - infrared: galaxies galaxies: fundamental parameters - galaxies: starburst

\section{Introduction}

Radio continuum and far infrared $(F I R)$ luminosities of starforming galaxies are known to show a tight linear relationship spanning four orders of magnitude in luminosity (van der Kruit 1973; De Jong et al. 1985; Condon 1992) and up to a redshift $\sim 1.3$ (Garrett 2002). This is interpreted as due to the presence of massive, young stars embedded in dust: a fraction of their UV radiation is absorbed by dust grains and reradiated in the infrared band, while supernova explosions may accelerate the electrons producing at radio wavelengths the observed synchrotron emission (Harwit \& Pacini 1975; Helou et al. 1985). Since massive $\left(M \gtrsim 5 M_{\odot}\right)$ stars are short-lived, these luminosities are assumed to be indicators of the global Star Formation Rate $(S F R)$ in a galaxy. Following Condon (1992) and Kennicutt (1998), the relation between $S F R$ (referred to stars with $M>5 M_{\odot}$ ) and radio/FIR luminosities can be written as:

$S F R=\frac{L_{1.4 \mathrm{GHz}}}{4.0 \times 10^{28}} M_{\odot} / \mathrm{yr}$

$S F R=\frac{L_{F I R}}{2.2 \times 10^{43}} M_{\odot} / \mathrm{yr}$

with the FIR flux defined after Helou et al. (1985) as:

$F I R=1.26 \times 10^{-11}\left(2.58 S_{60 \mu}+S_{100 \mu}\right) \mathrm{erg} \mathrm{s}^{-1} \mathrm{~cm}^{-2}$

where $L_{1.4 \mathrm{GHz}}$ is in $\mathrm{erg} \mathrm{s}^{-1} \mathrm{~Hz}^{-1}, L_{F I R}$ in $\mathrm{erg} \mathrm{s}^{-1}$ and infrared fluxes are in Jy.

Send offprint requests to: P. Ranalli, e-mail: ranalli@bo.astro.it
Star-forming galaxies are also luminous sources of X-ray emission, due to a number of High Mass X-ray Binaries (HMXB), young supernova remnants, and hot plasmas associated to star-forming regions and galactic winds (Fabbiano 1989). A non linear $\left(L_{X} \propto L_{F I R}^{0.6}\right)$ and much scattered (dispersion of about 2 dex) relation was found between $F I R$ and soft $(0.5-3.0 \mathrm{keV}) \mathrm{X}$-ray luminosities of IRAS-bright and/or interacting/peculiar galaxies measured by the Einstein satellite (Griffiths \& Padovani 1990). A somewhat different result was found by David et al. (1992), i.e. a linear relation between FIR and $0.5-4.5 \mathrm{keV}$ luminosities for a sample of starburst galaxies observed by Einstein. A large number of upper limits to the X-ray flux (12 upper limits vs. 11 detections for Griffiths \& Padovani 1990) along with high uncertainties in the X-ray and FIR fluxes may explain this discrepancy. Moreover, these studies suffered by the lack of knowledge about spectral shapes and internal absorption in star forming galaxies caused by the limited sensitivity and spectral capabilities of the IPC detector onboard Einstein.

Here, with the high sensitivity and the broad-band spectral capabilities of the ASCA and BeppoSAX satellites, we extend these studies to the $2-10 \mathrm{keV}$ band which is essentially free from absorption. In the following paragraphs a sample of nearby star forming galaxies is assembled (Sect. 2) and linear relations among radio, FIR and both soft and hard Xray luminosities are found (Sect. 3). Possible biases are discussed and the use of X-ray luminosities as a $S F R$ indicator is proposed (Sect. 4). In Sect. 5 we present a study of starforming galaxies in the Hubble Deep Field North and test the 
validity of the X-ray $S F R$ law. Implications for the contribution of star-forming galaxies to the X-ray counts and background are discussed in Sect. 6.

Throughout this paper, we assume $H_{0}=50$ and $q_{0}=0.1$.

\section{The local sample}

The atlas of optical nuclear spectra by Ho et al. (1997) (hereafter HFS97) represents a complete spectroscopic survey of galaxies in the Revised Shapley-Ames Catalog of Bright Galaxies (RSA; Sandage \& Tammann 1981) and in the Second Reference Catalogue of bright galaxies (RC2; de Vaucouleurs et al. 1976) with declination $\delta>0^{\circ}$ and magnitude $B_{T}<12.5$. Optical spectra are classified in HFS97 on the basis of line intensity ratios according to Veilleux \& Osterbrock (1987); galaxies with nuclear line ratios typical of star-forming systems are labeled as "H II nuclei". This sample of H II galaxies contains only spirals and irregulars from Sa to later types, except for a few S0 which were excluded from our analysis since their properties resemble more those of elliptical galaxies.

A cross-correlation of the HFS97 sample with the ASCA archive gives 18 galaxies clearly detected in the $2-10 \mathrm{keV}$ band with the GIS instruments. Four additional objects in the field of view of ASCA observations were not detected: the $2-10 \mathrm{keV}$ flux upper limits are too loose to add any significant information, and thus we did not include them in the sample. The crosscorrelation of the HFS97 sample with the BeppoSAX archive does not increase the number of detections. When a galaxy was observed by both satellites, we chose the observation with better quality data.

Far infrared fluxes at $60 \mu$ and $100 \mu$ were taken from the IRAS Revised Bright Galaxy Sample (RBGS, Mazzarella et al., in preparation) which is a reprocessing of the final IRAS archive with latest calibrations. While the RBGS measurements should be more accurate, we checked that the use of the older catalogue of IRAS observations of large optical galaxies ${ }^{1}$ by Rice et al. (1988), coupled with the Faint Source Catalogue (FSC, Moshir et al. 1989) for smaller galaxies, does not significantly change our statistical analysis. FIR fluxes for NGC 4449 were taken from Rush et al. (1993). Radio $(1.4 \mathrm{GHz})$ fluxes were obtained from the Condon et al. (1990, 1996) catalogues (except for NGC 4449, taken from Haynes et al. 1975). Distances were taken from Tully (1988) and corrected for the adopted cosmology.

Part of the X-ray data have already been published; in the cases where published data were not available in a form suitable for our analysis, the original data were retrieved from the archive and reduced following standard procedures and with the latest available calibrations. Images and spectra were extracted from the pipeline-screened event files. The images were checked against optical (Digital Sky Survey) and, where available, radio $(1.4 \mathrm{GHz})$ images in order to look for possible source confusions. Fluxes were calculated in the $0.5-2.0$ and 2-10 keV bands from best-fit spectra for the GIS2 and GIS3 instruments and corrected for Galactic absorption only. The uncertainty on the fluxes is of the order of $10 \%$. Depending on the

\footnotetext{
1 Blue-light isophotal major diameter $\left(D_{25}\right)$ greater than $8^{\prime}$.
}

quality of data, the best-fit spectrum is usually represented by a two-component model with a thermal plasma plus a power-law or just a power-law.

The galaxy IC 342 has shown some variability, mainly due to a burst in 1993 of an ultraluminous X-ray binary. M 82 is also a variable source. For each galaxy we summarize in the Appendix the results from several X-ray observations and estimate a time-averaged luminosity.

One object (M 33) was not included in the sample since its broad-band $(0.5-10 \mathrm{keV}) \mathrm{X}$-ray nuclear spectrum is dominated by a strong variable source (M $33 \mathrm{X}-8$ ) identified as a black hole candidate (Parmar et al. 2001).

Therefore, our sample (hereafter local sample) consists of the 17 galaxies listed in Table 1. Since it is not complete in a strict sense due to the X-ray selection, we have checked for its representativeness with reference to the $S F R$. The median $S F R$ values for HFS97 is $S F R_{\text {med }} \sim 1.65 M_{\odot} / \mathrm{yr}$. Considering objects with $S F R>S F R_{\text {med }}$ there are 14 galaxies in the local sample out of 98 in HFS97 (14\%), while there are 3 objects with $S F R<S F R_{\text {med }}(3 \%)$. Thus the high luminosity tail is better sampled than the low luminosity one.

We also include data for 6 other well-known starburst galaxies which were not in the HFS97 survey because they are in the southern emisphere. On the basis of their line intensity ratios $^{2}$ they should be classified as H II nuclei. In Table 1 we label them as supplementary sample.

\section{The radio/FIR/X-rays correlation}

As a preliminary test, we perform a least-squares analysis for the well-known radio/FIR correlation, which yields

$\log \left(L_{F I R}\right)=(0.98 \pm 0.06) \log \left(L_{1.4}\right)+15.4 \pm 1.6$.

The dispersion around the best-fit relation is given as the estimate $s$ of the standard deviation $\sigma$ :

$s=\frac{1}{N-v} \cdot \sqrt{\sum\left(\log L_{\mathrm{obs}}-\log L_{\mathrm{pred}}\right)^{2}}$

where $v$ is the number of free parameters, $N$ is the number of points in the fit, $L_{\text {pred }}$ is the luminosity expected from the best fit relation and $L_{\mathrm{obs}}$ the observed one. For the radio/FIR correlation (Eq. (4)) we find $s=0.18$.

Following Helou et al. (1985) we also calculate the mean ratio $q$ between the logarithms of $F I R$ and radio fluxes, obtaining $q \simeq 2.2 \pm 0.2$. This value is consistent with the mean $q=2.34 \pm 0.01$ for the 1809 galaxies in the IRAS 2 Jy sample by Yun et al. (2001).

\section{Soft X-rays}

A test for the soft X-ray/FIR/radio relations (Fig. 1) yields

$\log \left(L_{0.5-2}\right)=(0.87 \pm 0.08) \log \left(L_{F I R}\right)+2.0 \pm 3.7$

$\log \left(L_{0.5-2}\right)=(0.88 \pm 0.08) \log \left(L_{1.4}\right)+14.6 \pm 2.2$

with $s \simeq 0.26$ and 0.24 respectively.

\footnotetext{
${ }^{2}$ References: NGC 55 - Webster \& Smith (1983); NGC 253, 1672 \& 1808 - Kewley et al. (2001); NGC 3256 - Moran et al. (1999); Antennae - Rubin et al. (1970), Dobrodiy \& Pronik (1979).
} 
Table 1. Data for galaxies in our local samples. All galaxies were observed with ASCA, except those marked with * observed by BeppoSAX. Distances in Mpc; X-ray fluxes in $10^{-13} \mathrm{erg} \mathrm{s}^{-1} \mathrm{~cm}^{-2}, F I R$ fluxes in $10^{-9} \mathrm{erg} \mathrm{s}^{-1} \mathrm{~cm}^{-2}$ and radio fluxes in Jy; X-ray luminosities in $10^{40} \mathrm{erg} \mathrm{s}^{-1}$, FIR luminosities in $10^{43} \mathrm{erg} \mathrm{s}^{-1}$ and radio luminosities in $10^{29} \mathrm{erg} \mathrm{s}^{-1} \mathrm{~Hz}^{-1}$. The uncertainty on fluxes and luminosities is of the order of $10 \%$. References: 1. Cappi et al. (1999); 2. Della Ceca et al. (1996); 3. Della Ceca et al. (1999); 4. Zezas et al. (1998); 5. Della Ceca et al. (1997); 6. Dahlem et al. (1998); 7. Moran et al. (1999); 8. Sansom et al. (1996).

\begin{tabular}{|c|c|c|c|c|c|c|c|c|c|c|}
\hline \multicolumn{11}{|c|}{ Fluxes and Luminosities: Main Sample } \\
\hline GALAXY & $D$ & $F_{0.5-2}$ & $L_{0.5-2}$ & $F_{2-10}$ & $L_{2-10}$ & $F_{F I R}$ & $L_{F I R}$ & $F_{1.4}$ & $L_{1.4}$ & Refs. \\
\hline M 82* & 7.8 & 97 & 7.1 & 290 & 21 & 67 & 49 & 7.7 & 5.6 & 1 \\
\hline M 101 & 8.1 & 5.4 & 0.42 & 6.8 & 0.54 & 6.0 & 4.7 & 0.75 & 0.59 & this work \\
\hline M 108 & 21 & 4.4 & 2.3 & 6.0 & 3.2 & 2.0 & 11 & 0.31 & 1.63 & this work \\
\hline NGC 891 & 14 & 8.3 & 2.1 & 19 & 4.8 & 4.5 & 11 & 0.70 & 1.74 & this work \\
\hline NGC 1569 & 2.4 & 5.4 & 0.037 & 2.2 & 0.015 & 2.5 & 0.17 & 0.41 & 0.028 & 2 \\
\hline NGC 2146 & 26 & 8.2 & 6.5 & 11 & 9.0 & 7.3 & 59 & 1.1 & 8.7 & 3 \\
\hline NGC 2276 & 55 & 2.1 & 7.6 & 4.4 & 16 & 0.85 & 31 & 0.28 & 10 & this work \\
\hline NGC 2403 & 6.3 & 16 & 0.77 & 9.3 & 0.44 & 2.7 & 1.3 & 0.33 & 0.16 & this work \\
\hline NGC 2903 & 9.4 & 7.9 & 0.84 & 7.0 & 0.74 & 3.7 & 4.0 & 0.41 & 0.43 & this work \\
\hline NGC 3310 & 28 & 7.4 & 6.9 & 2.1 & 2.0 & 1.7 & 16 & 0.38 & 3.6 & 4 \\
\hline NGC 3367 & 64 & 1.8 & 8.7 & 1.6 & 7.9 & 0.38 & 19 & 0.10 & 4.9 & this work \\
\hline NGC 3690 & 69 & 5.7 & 33 & 11 & 62 & 5.3 & 310 & 0.66 & 38 & 4 \\
\hline NGC 4449 & 4.5 & 8.3 & 0.20 & 4.8 & 0.12 & 1.9 & 0.47 & 0.6 & 0.1 & 5 \\
\hline NGC 4631 & 10 & 9.4 & 1.2 & 9.3 & 1.2 & 4.9 & 6.2 & 1.2 & 1.5 & 6 \\
\hline NGC 4654 & 25 & 0.6 & 0.4 & 0.9 & 0.66 & 0.93 & 7.1 & 0.12 & 0.89 & this work \\
\hline NGC 6946 & 8.2 & 30 & 2.5 & 12 & 0.97 & 7.9 & 6.4 & 1.4 & 1.1 & this work \\
\hline IC 342 & 5.9 & 18 & 0.73 & 110 & 4.4 & 11 & 4.4 & 2.3 & 0.92 & this work \\
\hline \multicolumn{11}{|c|}{ SuPPLEMENTARY SAMPLE } \\
\hline NGC 55 & 1.9 & 18 & 0.082 & 6.8 & 0.031 & 4.7 & 0.21 & 0.38 & 0.017 & 6 \\
\hline NGC $253 *$ & 4.5 & 25 & 0.59 & 50 & 1.2 & 49 & 12 & 5.6 & 1.3 & 1 \\
\hline NGC 1672 & 22 & 5.8 & 3.2 & 6.1 & 3.4 & 2.3 & 13 & 0.45 & 2.5 & this work \\
\hline NGC 1808 & 16 & 6.5 & 2.0 & 7.6 & 2.4 & 5.3 & 17 & 0.52 & 1.6 & this work \\
\hline NGC 3256 & 56 & 9.0 & 34 & 6.2 & 23 & 4.8 & 180 & 0.66 & 25 & 7 \\
\hline Antennae & 38 & 7.2 & 13 & 5.3 & 9.2 & 2.6 & 45 & 0.57 & 9.9 & 8 \\
\hline
\end{tabular}

Our result is consistent with the $L_{0.5-4.5} \propto L_{F I R}^{0.95 \pm 0.06}$ relation found by David et al. (1992) for normal and starburst galaxies from the IRAS Bright Galaxy Sample, but it is only marginally consistent with the much flatter and more dispersed relationship obtained by Griffiths \& Padovani (1990) for a sample of IRAS selected galaxies $\left(L_{0.5-3 \mathrm{keV}} \propto L_{60 \mu}^{0.62 \pm 0.14}\right)$ and for a sample of starburst/interacting galaxies $\left(L_{0.5-3 \mathrm{keV}} \propto L_{60 \mu}^{0.70 \pm 0.12}\right)$.

The inclusion of the objects of the supplementary sample (Table 1) does not significantly change the slopes, i.e. $L_{0.5-2.0 \mathrm{keV}} \propto L_{F I R}^{0.88 \pm 0.07}$; likewise, if we use the $60 \mu$ luminosity instead of $F I R$, we obtain $L_{0.5-2.0 \mathrm{keV}} \propto L_{60 \mu}^{0.85 \pm 0.07}$.

By assuming an exactly linear slope, the best fit relations for the local (local+supplementary) sample become:

$\log \left(L_{0.5-2}\right)=\log \left(L_{F I R}\right)-3.68(3.70)$

$\log \left(L_{0.5-2}\right)=\log \left(L_{1.4}\right)+11.08$ (11.10)

with $s \simeq 0.27$ and 0.24 respectively.

By applying an F-test we find that the free-slope fits are not significantly better than those with the linear slope, the improvement being significant only at the $1 \sigma$ level.

\section{Hard X-rays}

In Fig. 2 we plot 2-10 keV luminosities versus FIR and radio ones. Least-squares fits yield:

$\log \left(L_{2-10}\right)=(1.08 \pm 0.09) \log \left(L_{F I R}\right)-7.1 \pm 4.2$

$\log \left(L_{2-10}\right)=(1.08 \pm 0.09) \log \left(L_{1.4}\right)+8.8 \pm 2.7$

with $s \simeq 0.30$ and 0.29 respectively. The linearity and the dispersion are not significantly changed neither by the inclusion of the supplementary sample $\left(L_{2-10} \propto L_{F I R}^{1.04 \pm 0.07}\right.$ and $L_{2-10} \propto$ $L_{1.4}^{1.01 \pm 0.07}, s \simeq 0.27$ and 0.26 respectively), nor by the use of the $60 \mu$ luminosity $\left(L_{2-10} \propto L_{60 \mu}^{1.01 \pm 0.07}\right)$.

By assuming an exactly linear slope, the best fit relations for the local (local+supplementary) sample become:

$$
\begin{aligned}
& \log \left(L_{2-10}\right)=\log \left(L_{F I R}\right)-3.62(3.68) \\
& \log \left(L_{2-10}\right)=\log \left(L_{1.4}\right)+11.13(11.12)
\end{aligned}
$$

with $s \simeq 0.29$ for both fits. There is no significant improvement (less than $1 \sigma$ ) in the free-slope fits with respect to the linear slope ones.

\section{X-rays and the Star Formation Rate}

The existence of a tight linear relation implies that the three considered bands all carry the same information. Since the 

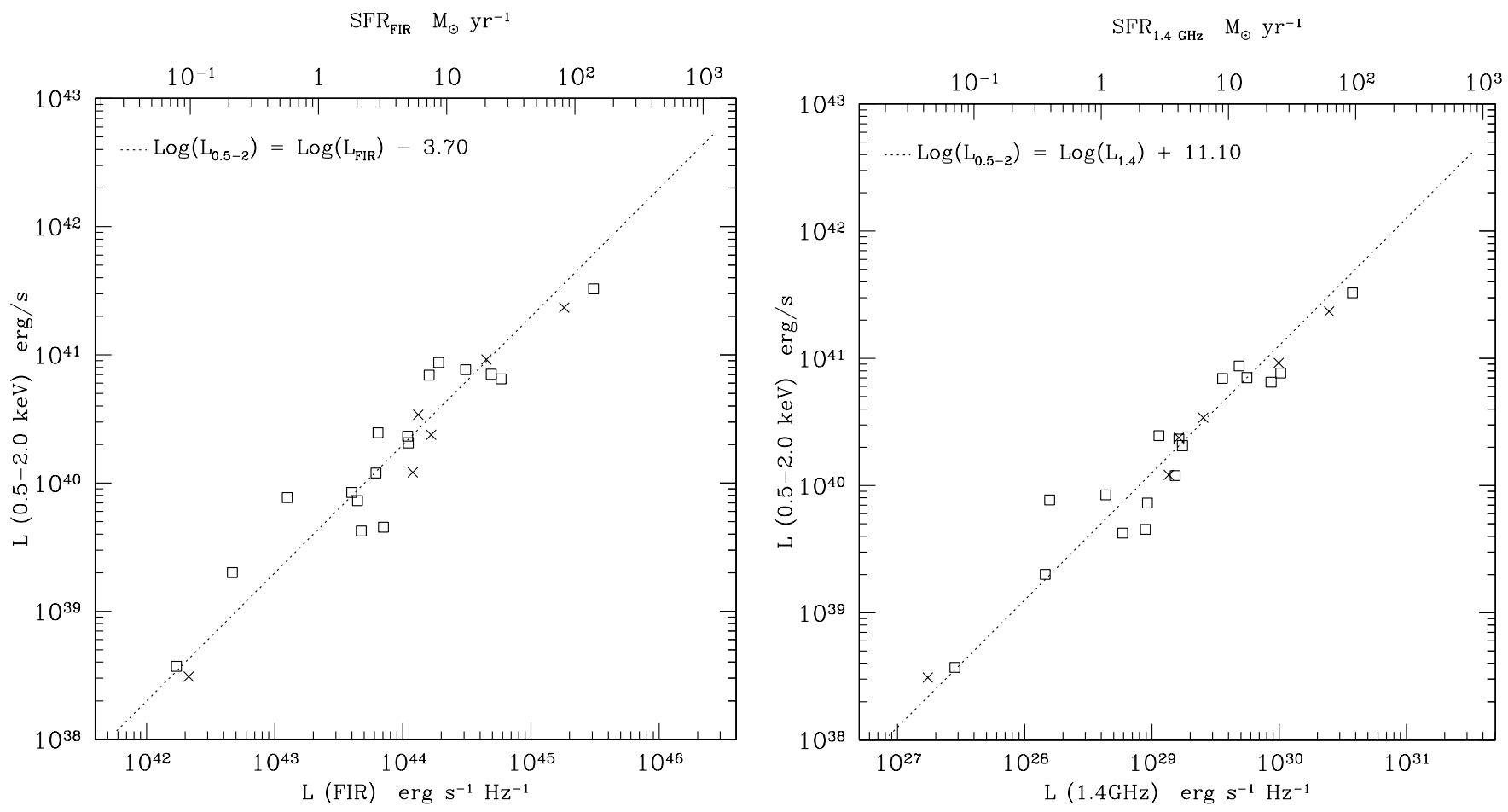

Fig. 1. The $0.5-2.0 \mathrm{keV}$ luminosity of local star-forming galaxies vs. radio and FIR ones. Squares: local sample; crosses: supplementary sample; dotted lines: Eqs. (8), (9).
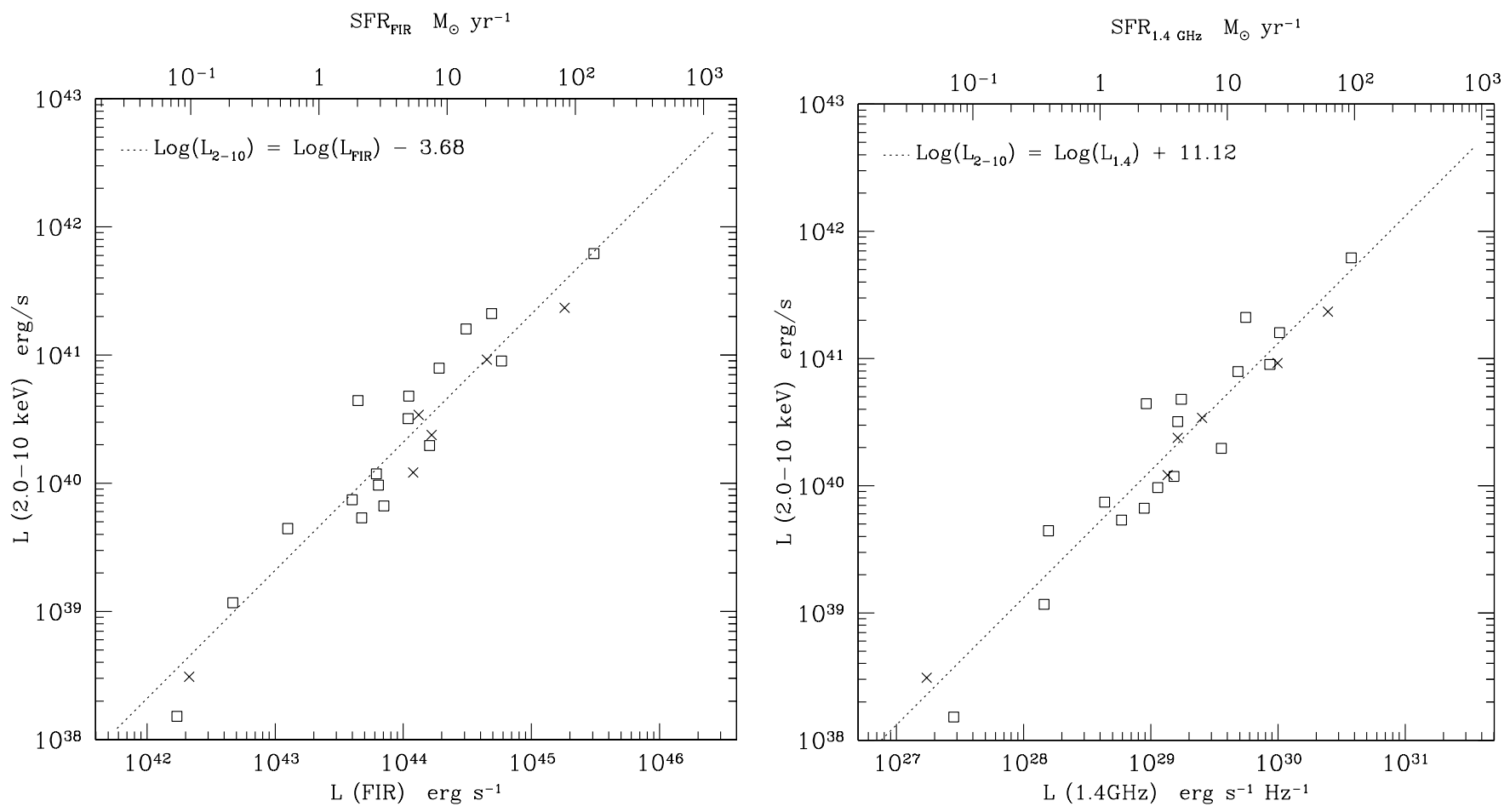

Fig. 2. The 2.0-10 keV luminosity of local star-forming galaxies vs. radio and FIR ones. Symbols as in Fig. 1; dotted lines: Eqs. (12), (13).

radio and far infrared luminosities are indicators of the $S F R$, the $0.5-2 \mathrm{keV}$ and $2-10 \mathrm{keV}$ luminosities should also be $S F R$ indicators. However, before attempting to calibrate such relationships, we should consider the possible existence of selection effects.

Ho et al. (1995) made a special effort in obtaining $n u$ clear nebular spectra, so that a reliable spectral classification of the central engine could be derived. The main concern is the possibility that the H II galaxies in the HFS97 sample could host a Low Luminosity AGN (LLAGN), which might significantly contribute to the overall energy output. To check for this possibility, Ulvestad \& Ho (2002) observed with the VLA at $1.4 \mathrm{GHz}$ a complete sample of $40 \mathrm{Sc}$ galaxies in HFS97 with $\mathrm{H}_{\text {II }}$ spectra and did not find any compact luminous radio 


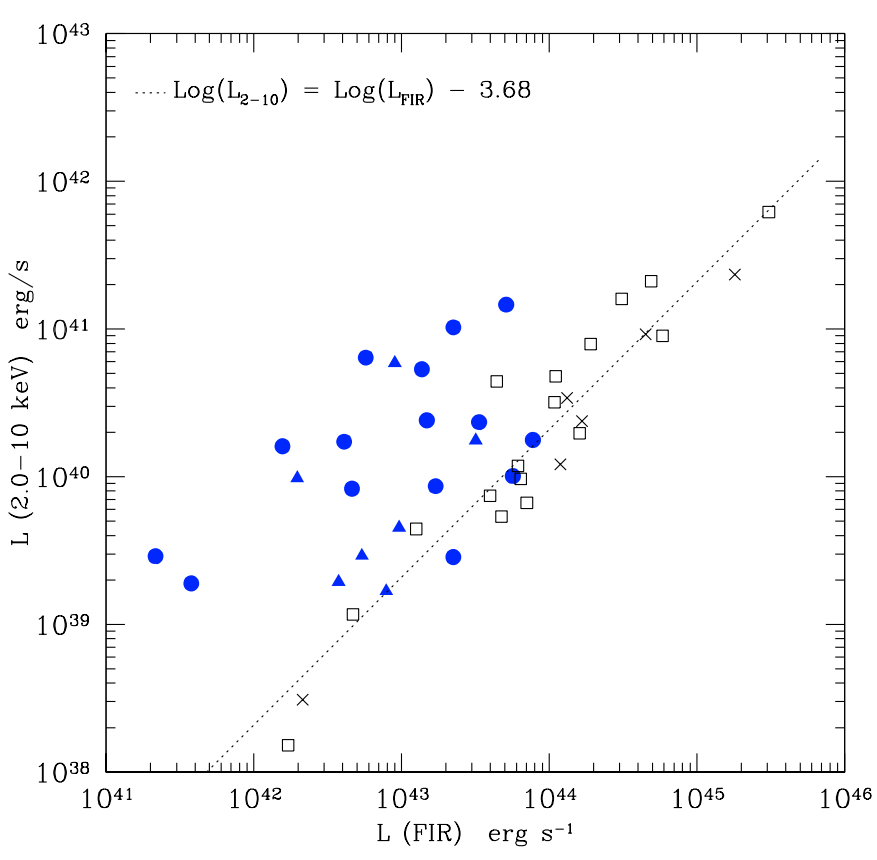

Fig. 3. $2.0-10 \mathrm{keV}$ vs. FIR luminosities of star-forming galaxies and LLAGN. Filled triangles: LINERs, filled circles: Seyfert galaxies. Open symbols as in Fig. 1; line: Eq. (12).

core. Instead, they found that the radio powers and morphologies are consistent with star formation processes rather than by accretion onto massive black holes; thus they suggest that H II nuclei intrinsically lack AGN. Therefore we believe that the HFS97 classification is reliable and that our sample is not polluted by AGN.

It is also worth noticing that the soft X-rays relationships may involve some further uncertainties related to the possible presence of intrinsic absorption (negligible in the $2-10 \mathrm{keV}$ band for column densities usually found in normal galaxies). An example of this effect is the southern nucleus of NGC 3256 (see the Appendix), a dusty luminous merger remnant with two bright radio-IR cores where star formation is ongoing: while both of them fall on the radio/hard X-ray relation, only the northern core is on the radio/soft X-ray relation because the southern one lies behind a dust lane which absorbes at all wavelengths from $\sim 1 \mu$ to $\sim 2 \mathrm{keV}$. The quasi-linearity of the soft $\mathrm{X}$-ray relations suggests that absorption is unlikely to be relevant for the majority of the objects in our sample; however this effect may become significant at cosmological distances $(z \gtrsim 1-2)$ where galaxies have more dust and gas at their disposal to form stars.

Thus we feel confident to propose the use of X-ray luminosities as SFR indicators. From Eqs. (8), (9), (12), (13) we derive:

$$
\begin{array}{ll}
S F R=2.2 \times 10^{-40} L_{0.5-2 \mathrm{keV}} & M_{\odot} / \mathrm{yr} \\
S F R=2.0 \times 10^{-40} L_{2-10 \mathrm{keV}} & M_{\odot} / \mathrm{yr} .
\end{array}
$$

We also notice that there is growing evidence that star formation could play a major role even among those objects classified as LLAGN. The preliminary results of the Chandra LLAGN survey (Ho et al. 2001) show that only about one third of
Table 2. Identification of candidate star-forming galaxies in the Richards et al. (1998) catalogues with their X-ray counterpart (Brandt et al. 2001). The entry number in the Chandra catalogue is shown in parenthesis.

\begin{tabular}{cc}
\hline \hline CHANDRA & VLA \\
\hline CXOHDFN J123634.4+621212 (134) & $3634+1212$ \\
CXOHDFN J123634.5+621241 (136) & $3634+1240$ \\
CXOHDFN J123637.0+621134(148) & $3637+1135$ \\
CXOHDFN J123651.1+621030(188) & $3651+1030$ \\
CXOHDFN J123653.4+621139(194) & $3653+1139$ \\
CXOHDFN J123708.3+621055 (246) & $3708+1056$ \\
CXOHDFN J123716.3+621512(278) & $3716+1512$ \\
\hline
\end{tabular}

LLAGN have a compact nucleus dominating the X-ray emission, while in the remaining objects off-nuclear sources and diffuse emission significantly contribute to the overall emission.

Following this investigation, we have analyzed the relations between radio/FIR/X-ray luminosities for the spiral galaxies in the Terashima et al. (2002) sample of LLAGN, drawn from HFS97 and observed with ASCA, comprising 7 LINERs and 15 Seyfert's with $4 \times 10^{39} \lesssim L_{2-10} \lesssim 5 \times 10^{41} \mathrm{erg} \mathrm{s}^{-1}$. We find that the $\mathrm{X}$-ray/FIR and $\mathrm{X}$-ray/radio luminosity ratios generally exceed those of star-forming galaxies, but about one third of the objects have ratios falling on the same locus of the star-forming galaxies (Fig. 3). Therefore, the nuclear X-ray emission of these last LLAGN must be comparable to or weaker than the emission from star formation related processes. Moreover, the infrared (IRAS band) colours of these objects are also similar to those of star-forming galaxies, and completely different from those of QSOs, thus suggesting that the FIR luminosities of LLAGN may be powered by star formation.

\section{Star-forming galaxies in the Hubble Deep Field}

The 1 Ms Chandra (Brandt et al. 2001) and the radio (8.4 GHz: Richards et al. 1998; 1.4 GHz: Richards 2000 and Garrett 2000) catalogues of the Hubble Deep Field North (HDFN) reach a limiting flux which is deep enough to detect star-forming galaxies at redshifts up to $z \sim 1.3$, and can be used to check whether the radio/X-ray relation holds also for distant galaxies.

We searched for X-ray counterparts of radio sources in the the Richards et al. (1998) catalogue which contains optical and IR identifications allowing the selection of candidate starforming galaxies. Our selection criterium has been to include all galaxies with Spiral or Irregular morphologies, known redshifts and no AGN signatures in their spectra (from Richards et al. 1998 or Cohen et al. 2000).

The mean positional uncertainties of both Chandra (for onaxis sources) and the VLA are $\sim 0.3^{\prime \prime}$, which added in quadrature give $\sim 0.5^{\prime \prime}$. Using this value as the encircling radius for coordinate matching 5 galaxies are found. However, there are two effects that can increase this value:

1. the shape and width of the Chandra PSF strongly depend on the off-axis and azimutal angles. Since the 1 Ms HDFN data consist of 12 observations with different pointing directions and position angles, there is no unique PSF model even for near on-axis sources; 


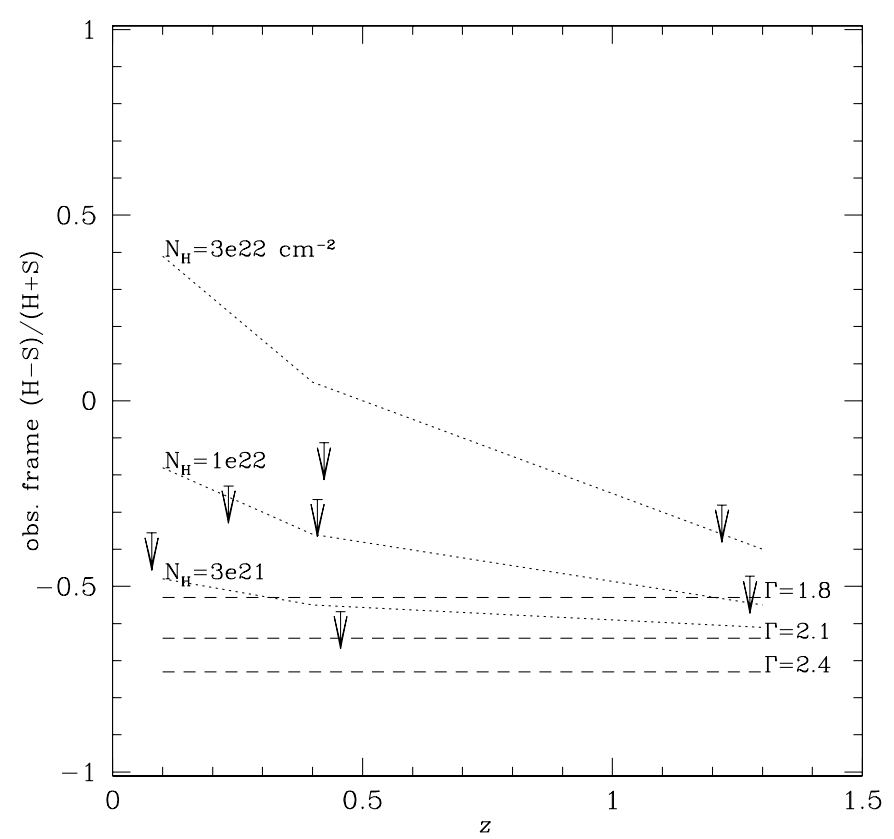

Fig. 4. Observer's-frame hardness ratios for galaxies in the HDFN; there are only upper limits since none is detected in the $2-8 \mathrm{keV}$ band (Brandt et al. 2001). The dotted lines show the loci for power-law spectra with slope $\Gamma=2.1$ and different intrinsic rest-frame absorption; the dashed lines are for spectra of different slopes and no intrinsic absorption.

2. a displacement of several kpc between the brightest radio and X-ray positions, induced e.g. by an ultraluminous Xray binary placed in a spiral arm and dominating the X-ray emission.

Thus, by making cross-correlations with increasing encircling radii we found that the number of coincidences increases up to a radius of $1.0^{\prime \prime}$ (which yields 7 matchings). There are no further coincidences up to a radius of several arcsecs, indicating that the sample should not be contamined by chance coincidences. The 7 selected objects are listed in Table 2. Fluxes at $1.4 \mathrm{GHz}$ and spectral slopes were retrieved from Richards (2000) in 6 cases, and from Garrett (2000) in one case.

While rest-frame $0.5-2.0$ and $2.0-10 \mathrm{keV}$ luminosities could be obtained by $K$-correcting the observer's frame counts, this would imply the assumption of a spectral shape; but none of the seven deep field galaxies is individually detected in the hard band, so that any constraint on their spectra obtained with the use of a hardness ratio diagram is too loose to be significant (Fig. 4). However this problem may be partially circumvented by resizing the X-ray bands in the observer's frame according to the redshift of the objects. It is therefore possible to give better constraints to the spectrum of the deep field galaxies and derive better estimates of their luminosities.

Thus we redefine the soft and hard ${ }^{3}$ bands as the $[0.5$; $2.0 /(1+z)]$ and $[2.0 /(1+z) ; 10 /(1+z)]$ intervals, respectively.

\footnotetext{
${ }^{3}$ Since Chandra has very poor sensitivity between 8 and $10 \mathrm{keV}$, the use of the reduced $2-8 \mathrm{keV}$ band enhances the signal/noise ratio. Note that while counts are extracted in the $2-8 \mathrm{keV}$ band, fluxes and luminosities are always extrapolated to the $2-10 \mathrm{keV}$ band.
}

Another advantage of this procedure is that the higher the redshift, the more akin the new hard band is to the zone of maximum sensitivity of Chandra $(\sim 1-4 \mathrm{keV})$. Note that since the ACIS-I detector has almost no sensitivity below $0.5 \mathrm{keV}$, we fixed this energy as the lower limit for count extraction. For the two highest redshift galaxies, this reduces the soft band to 0.5-0.9 keV, still significantly larger than the ACIS-I energy resolution $(F W H M \lesssim 100 \mathrm{eV})$.

We extracted counts in circular regions around our selected targets (radius 5 pixels); background was taken in annuli surrounding the targets. The net counts did not show dependence on the choice of background regions; they were converted in count rates with the exposure times listed in the Brandt et al. (2001) catalogue. Counts and rates are reported in Table 3. Best-fit slopes reproducing the soft/hard count ratio were derived by assuming a power-law spectrum with Galactic absorption. We find that six objects have spectral slopes falling in the range $\Gamma \sim 1.5-2.7$ (Table 3 ). To check whether these spectra are consistent with those of the galaxies in the local sample we calculated the observed soft/hard flux ratio for galaxies in the local sample: the median value for this flux ratio is 0.95 leading to a slope $\Gamma \sim 2.1$. The count ratios for each of the six deep field galaxies are consistent within $1-2 \sigma$ with the $\Gamma \sim 2.1$ slope. The remaining source (\#194 in Table 3, at $z=1.275$ ) has an upper limit on the soft X-ray counts and a count ratio not consistent at the $5 \sigma$ level with the unabsorbed $\Gamma \sim 2.1$ spectrum: it requires an inverted spectrum $(\Gamma<0.1)$ if no absorption is assumed, otherwise, if we assume $\Gamma=2.1$, the intrinsic absorbing column has to be $N_{\mathrm{H}} \gtrsim 3.4 \times 10^{22} \mathrm{~cm}^{-2}$.

With the best-fit slopes we derived soft and hard band fluxes and luminosities (Fig. 5). The linear radio/X-ray correlations hold also for the Deep Field galaxies; the dispersion of the relations given in Sect. 3 is not changed by the inclusion of the deep field objects. A similar relation was also found in a recent paper by Bauer et al. (2002).

\section{X-ray number counts and background}

An estimate of the contribution of star-forming galaxies to the cosmic X-ray background (XRB) has been attempted several times (e.g. Bookbinder et al. 1980; Griffiths \& Padovani 1990; Moran et al. 1999). The main purpose for the earlier studies was the possibility to explain the flatness of the XRB spectrum via the X-ray binaries powering the X-ray emission of these galaxies. Although AGN have since been recognized to provide by far the most important contribution to the XRB (Setti \& Woltjer 1989; Comastri et al. 1995), the ongoing deep Chandra and XMM-Newton surveys offer unique opportunities to both test the AGN models and pin down the contribution from other kind of sources. Here, making use of the X-ray/radio relationships of Sect. 3, we perform an estimate of the contribution to the XRB by star-forming galaxies.

We consider the radio sub-mJy population associated with faint blue galaxies at high redshifts $(22 \lesssim V \lesssim 27,0.5 \lesssim$ $z \lesssim 1.5$; Windhorst et al. 1990) representing an early era of star formation in the universe (Haarsma et al. 2000). This strongly evolving population accounts for the majority of the number counts below $\sim 0.5 \mathrm{mJy}$ (Windhorst et al. 1985) and 

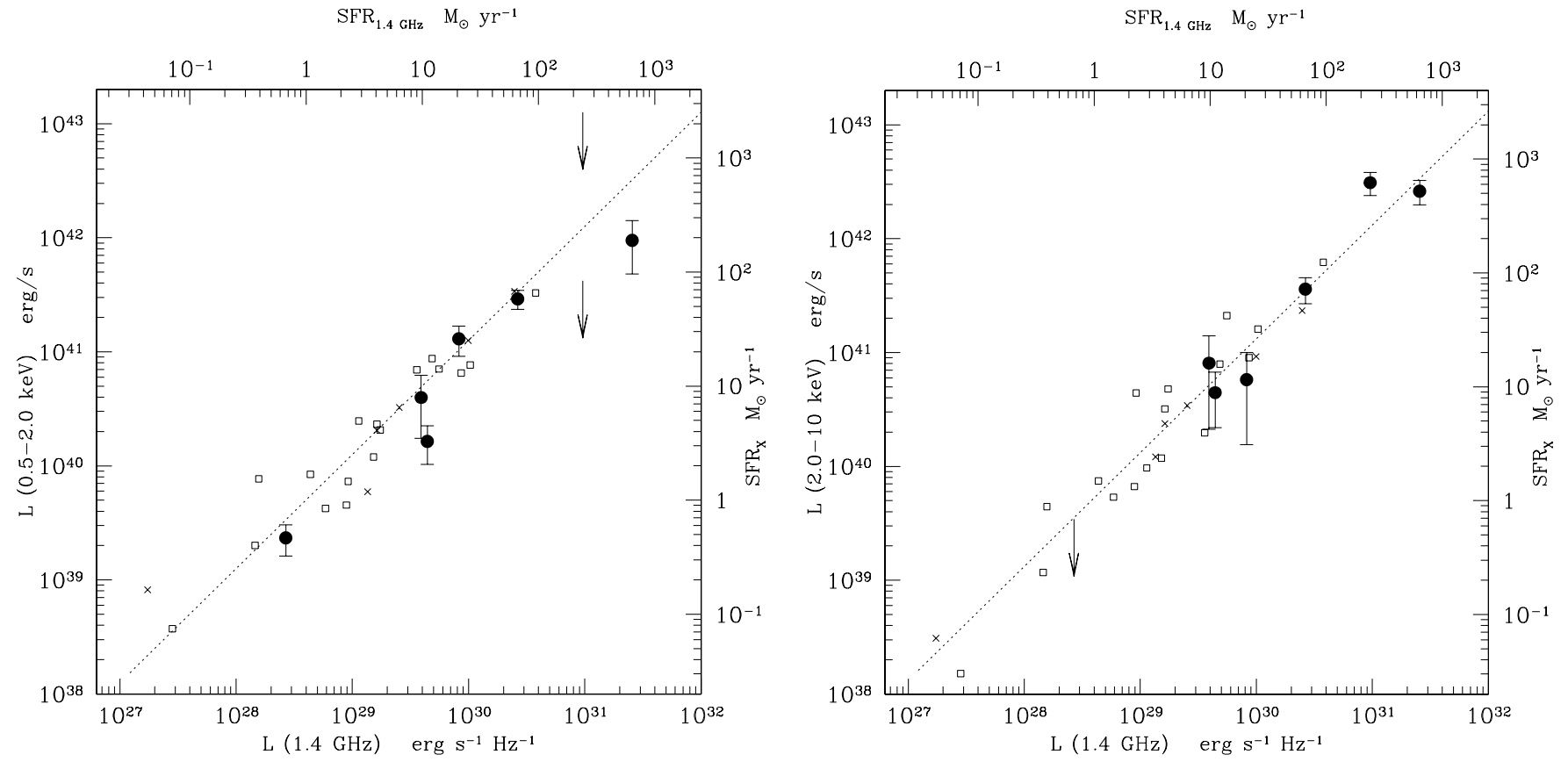

Fig. 5. The radio/X-ray luminosity relation for galaxies in the Hubble Deep Field (filled circles). Open squares and crosses: local galaxies as in Fig. (1); dotted lines: linear fits for local galaxies (Eq. (9)). The two most luminous galaxies are at $z=1.275$ and 1.219 in order of increasing radio luminosity. The two upper limits ( $3 \sigma$ level) in the left panel refer to source \#194 (see text) and show the unabsorbed luminosity as estimated with $\Gamma \sim 0.1$ and no intrinsic absorption (lower) and with $\Gamma \sim 2.1$ and $N_{\mathrm{H}} \sim 3.4 \times 10^{22} \mathrm{~cm}^{-1}$ (upper).

contribute about half of the radio cosmic background at $1.4 \mathrm{GHz}$ (Haarsma et al. 2000). The deepest radio surveys have been performed at $1.4 \mathrm{GHz}$ (Richards 2000), $5 \mathrm{GHz}$ (Fomalont et al. 1991) and $8.4 \mathrm{GHz}$ (Richards et al. 1998). Although a quick estimate of the contribution to the XRB may be worked out by simply applying Eqs. (9), (13) to the total radio fluxes obtained by integrating over the deepest radio $\log N-\log S$, it is instructive to first derive the X-ray source counts and compare them with the deepest counts obtained by Chandra. In order to derive the X-ray counts from the radio ones a full knowledge of the redshift distribution and spectra of the sources would be required. Under the simplifying assumption that the sub-mJy population lies at a redshift $\bar{z}$, so that the $K$-correction term is the same for all sources, the differential counts are obtained as:

$n\left(S_{\mathrm{X}}\right)=n\left(S_{1.4} \cdot \kappa_{1.4}^{\mathrm{X}}\right)$

where $n(S)$ are the differential number counts, and $\kappa_{1.4}^{\mathrm{X}}=$ $\kappa_{1.4}^{\mathrm{X}}(\bar{z}, \alpha, \Gamma)$ is the $\mathrm{X}$-ray band $/ 1.4 \mathrm{GHz}$ luminosity ratio (Eqs. (9), (13)) which depends on the redshift and on the radio and $\mathrm{X}$-ray spectral indices via the $K$-correction:

$\kappa_{1.4}^{\mathrm{X}}(\bar{z}, \alpha, \Gamma)=\left.\kappa_{1.4}^{\mathrm{X}}\right|_{z=0} \cdot(1+\bar{z})^{-(\Gamma-1)+\alpha}$.

Concerning the sub-mJy $\log N-\log S$, Richards (2000) gives $n(S)=(2.51 \pm 0.13) \times 10^{-3} S^{-2.38 \pm 0.13} \mathrm{deg}^{-2} \mathrm{Jy}^{-1}$ as a best-fit to the differential number counts at $1.4 \mathrm{GHz}$ in the range $45-1000$, while Fomalont et al. (1991) find $n(S)=1.2 \times 10^{-3} S^{-2.18 \pm 0.19}$ at $5 \mathrm{GHZ}$ in the range $16-1500 \mu \mathrm{Jy}$. The number density at $4 \mu \mathrm{Jy}$ (as estimated from fluctuation analysis) is consistent with extrapolation of the $16-1500 \mu \mathrm{Jy}$ slope.

The mean radio spectral index is in the range $\alpha=0.3$ 0.7. Fomalont et al. (1991) report their distribution as having a moda of $\alpha=0.5$, a median of $\alpha=0.38$, and an average of $\alpha=0.28$ (indices measured between 1.5 and $5 \mathrm{GHz}$ ). From Richards (2000) data we find an average $\alpha=0.47$ (between 1.4 and $8.4 \mathrm{GHz}$ ), when we consider detections at both frequencies, and $\alpha=0.67$ when we consider only detections at 1.4 GHz and treat upper-limits at $8.4 \mathrm{GHz}$ as detections. The latter, steeper slope is also more consistent with the average index of our HDFN sample (Table 3). Here we assume $\alpha=0.5$, and estimate the uncertainty in the $K$-correction due to the radio spectral index to be around a 15-20\%.

According to the results of Sect. 5, we further assume an average X-ray spectral slope $\Gamma=2.1$. For the sake of simplicity we also assume that the objects are placed at $\bar{z} \sim 1$, the mean redshift for the sub-mJy galaxies which, according to Windhorst et al. (1990), are distributed in the redshift interval $0.5-1.5$ with a peak at $z \sim 1$. To estimate the effect on the counts due to the actual distribution of the sources in this redshift interval we consider a simplified case in which they are equally distributed at redshifts $z=0.5,1.0$ and 1.5. Since the effect enters in the computations via the $K$-correction term, we find that the predicted number counts would increase by only $\sim 10 \%$. As a last remark we notice that a fraction, as yet undefined, of the X-ray spectra might steepen at energies $\gtrsim 10 \mathrm{keV}$, thus entailing a decrease in the predicted source counts; however, given the redshift range under consideration, the predicted soft X-ray source counts should not be affected.

The X-ray number counts predicted with the above assumptions (i.e. $\alpha=0.5, \Gamma=2.1$ ) from Richards (2000) and Fomalont et al. (1991) Log $N-\log S$ are shown in Fig. 6, along with observed counts from Brandt et al. (2001), limits from fluctuation analysis (Miyaji \& Griffiths 2002a,b) and predicted 
Table 3. Data for deep field galaxies (counts, fluxes, luminosities). $\Gamma$ is the best fit $X$-ray slope (photon index), $\alpha$ the radio slope (energy index). Sources are identified via their entry number in the Brandt et al. (2001) catalogue (cf. Table 2). X-ray fluxes in $10^{-17} \mathrm{erg} \mathrm{s}^{-1} \mathrm{~cm}^{-2}$, radio fluxes in $\mu \mathrm{Jy}$. X-ray luminosities in $10^{40} \mathrm{erg} \mathrm{s}^{-1}$, radio luminosities in $10^{30} \mathrm{erg} \mathrm{s}^{-1} \mathrm{~Hz}^{-1}$. X-ray counts are extracted in redshifted bands (soft band $:=0.5-2.0 /(1+z) \mathrm{keV}$, hard band $:=2.0 /(1+z)-8 /(1+z) \mathrm{keV})$. X-ray fluxes and luminosities are in rest-frame $0.5-2.0$ and $2.0-10 \mathrm{keV}$ bands, radio ones at rest-frame $1.4 \mathrm{GHz}$. For source \#194, the absorbed flux and luminosity are quoted. The unabsorbed luminosity is $\lesssim 4 \times 10^{42} \mathrm{erg} \mathrm{s}^{-1}$.

\begin{tabular}{|c|c|c|c|c|c|c|c|c|c|c|c|}
\hline \multicolumn{12}{|c|}{ FluXes and Luminosities: DeEP SAMPLE } \\
\hline \multirow[b]{2}{*}{ SOURCE } & \multirow[b]{2}{*}{$z$} & \multicolumn{3}{|c|}{ SOFT X-RAYS } & \multicolumn{3}{|c|}{ HARD X-RAYS } & \multirow[b]{2}{*}{$\Gamma$} & \multicolumn{3}{|c|}{ RADIO } \\
\hline & & Counts & $F$ & $L$ & Counts & $F$ & $L$ & & $F$ & $L$ & $\alpha$ \\
\hline 134 & 0.456 & $31 \pm 6$ & 23 & 29 & $22 \pm 6$ & 28 & 36 & $2.0_{-03}^{+0.4}$ & 210 & 2.6 & 0.7 \\
\hline 136 & 1.219 & $5.9 \pm 3$ & 6.7 & 95 & $22 \pm 5$ & 19 & 260 & $1.6_{-0.5}^{+0.9}$ & 180 & 26 & 0.7 \\
\hline 148 & 0.078 & $14 \pm 4$ & 8.4 & 0.23 & $<2.1$ & $<4.1$ & $<0.11$ & $>2.6$ & 96 & 0.027 & 0.6 \\
\hline 188 & 0.410 & $15 \pm 4$ & 13 & 13 & $5.2 \pm 4$ & 5.8 & 5.8 & $2.7_{-0.6}^{+1.5}$ & 83 & 0.82 & 0.6 \\
\hline 194 & 1.275 & $<1.6$ & $<0.88$ & $<14$ & $23 \pm 5$ & 20 & 310 & $<0$ & 60 & 9.6 & 0.9 \\
\hline 246 & 0.423 & $6.2 \pm 4$ & 3.7 & 4.0 & $5.4 \pm 4$ & 7.5 & 8.1 & $1.7_{-06}^{+1.4}$ & 36 & 0.39 & 0.4 \\
\hline 278 & 0.232 & $11 \pm 4$ & 5.8 & 1.6 & $7.8 \pm 4$ & 16 & 4.4 & $\begin{array}{l}1.5_{-0.0}^{+0.0} \\
\end{array}$ & 160 & 0.44 & 0.2 \\
\hline
\end{tabular}
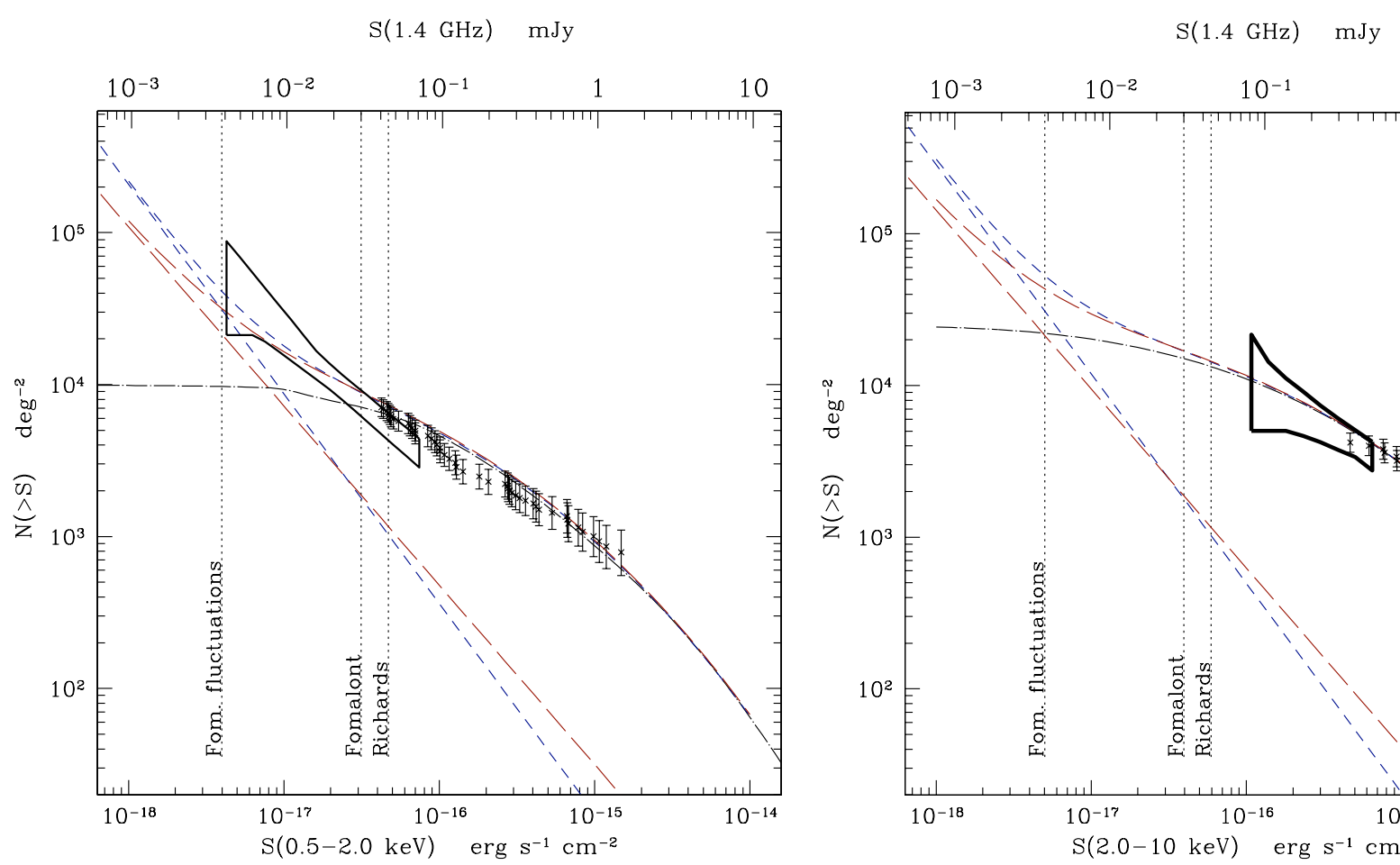

Fig. 6. X-ray counts derived from deep radio $\log N$-Log $S$. The short-dashed lines represent the $1.4 \mathrm{GHz} \log N-\log S$ (Richards 2000 ) with $K$-corrections assuming $\bar{z}=1, \Gamma=2.1$ and $\alpha=0.5$. The long-dashed lines represent the $\log N-\log S$ by Fomalont et al. (1991) reported at $1.4 \mathrm{GHz}$ with $\alpha=0.5$ ( $K$-corrections like for the $1.4 \mathrm{GHz}$ one). The dots are the observed X-ray number counts in the HDFN (Brandt et al. 2001); while the horn-shaped symbols show the results from X-ray fluctuation analysis (Miyaji \& Griffiths 2002a,b). The dot-dashed line shows the number counts from AGN synthesis models (Comastri et al. 1995). The vertical dotted lines show the limiting sensitivities for the radio surveys. The total of AGN plus galaxy counts predicted from the radio counts of Richards (2000) and Fomalont et al. (1991) are also shown with short and long dashed lines, respectively.

counts from AGN synthesis models (Comastri et al. 1995). The latter are essentially in agreement with the results of more recent synthesis models (Gilli et al. 2001). The extrapolations of the radio $\log N-\log S$ below $\sim 50 \mu \mathrm{Jy}$ and down to a few $\mu \mathrm{Jy}$ at $1.4 \mathrm{GHz}\left(\sim 6 \times 10^{-18} \mathrm{erg} \mathrm{s}^{-1} \mathrm{~cm}^{-2}\right.$ in the $0.5-2.0 \mathrm{keV}$ band $)$ are consistent with the limits from fluctuation analysis (Miyaji \& Griffiths 2002b) and do not exceed the X-ray expected number counts. Since Miyaji \& Griffiths (2002b) suggested the emergence of a new population (beyond that of AGN which dominates at brighter fluxes) in the $0.5-2.0 \mathrm{keV}$ band at fluxes around $10^{-17} \mathrm{erg} \mathrm{s}^{-1} \mathrm{~cm}^{-2}$, it is tempting to identify this new population with the sub-mJy galaxies. It should be noted, however, that Eqs. (9), (13) may not apply to the entire sub-mJy population. For instance, a fraction (up to a $15-20 \%$, Haarsma et al. 2000) of the sources may still present a dominant contribution from an AGN. It follows that the derived $\log N-\log S$ should be regarded as an upper limit to the $\mathrm{X}$-ray counts from star-forming galaxies. 
As a final remark it should be pointed out that our results depend on the basic assumption of a strict linearity between the radio and $\mathrm{X}$-ray luminosities. Had we assumed a non-linear relationship, such as Eq. (7) for the soft X-rays or Eq. (11) for the $2-10 \mathrm{keV}$ band, we would have found an increase or decrease, respectively, of about $50 \%$ in the predicted counts at a flux level around $10^{-17} \mathrm{erg} \mathrm{s}^{-1} \mathrm{~cm}^{-2}$. A larger, well defined sample of star-forming galaxies down to the faintest radio and $\mathrm{X}$-ray fluxes would obviously be of great importance to better constrain the $\mathrm{X}$-ray vs. radio luminosities relationship. It might be argued that a more direct derivation of the source counts could be made by adopting the observed fluxes, rather than the luminosities of the objects. However, this procedure would entail an arbitrary extrapolation of the X-ray/radio flux ratio for almost two orders of magnitude at fluxes fainter than the Chandra deep field. On the other hand, the radio luminosity interval of Fig. 5 essentially encompasses the radio power of the sub-mJy population if placed in the redshift interval mentioned above.

The derived X-ray number counts can be integrated to estimate the contribution to the XRB. For the observed 2$10 \mathrm{keV}$ background we take the XMM-Newton value of $2.15 \times$ $10^{-11} \mathrm{erg} \mathrm{s}^{-1} \mathrm{~cm}^{-2}$ (Lumb et al. 2002), which is comprised between the ASCA (Gendreau et al. 1995) and BeppoSAX (Vecchi et al. 1999) figures. The integration of counts derived from the $1.4 \mathrm{GHz} \log N-\log S$ (Richards 2000), performed in its validity range $\left(5.9 \times 10^{-17}-1.3 \times 10^{-15} \mathrm{erg} \mathrm{s}^{-1} \mathrm{~cm}^{-2}\right.$, corresponding to $45-1000 \mu \mathrm{Jy}$ at $1.4 \mathrm{GHz}$ ), yields a contribution to the XRB of $4.0 \times 10^{-13} \mathrm{erg} \mathrm{cm}^{-2} \mathrm{~s}^{-1}$ ( $\$ 2 \%$ of the observed background). By extrapolating to $10^{-18} \mathrm{erg} \mathrm{s}^{-1} \mathrm{~cm}^{-2}(\sim 1 \mu \mathrm{Jy})$ the contribution would increase to $2.3 \times 10^{-12} \mathrm{erg} \mathrm{cm}^{-2} \mathrm{~s}^{-1}$ $(11 \%)$. Integration of counts from the flatter Fomalont et al. (1991) $\log N-\log S$ in the $1-1000 \mu \mathrm{Jy}$ range yields a contribution of $1.4 \times 10^{-12} \mathrm{erg} \mathrm{cm}^{-2} \mathrm{~s}^{-1}(6.4 \%)$. We also note that $1 \mu \mathrm{Jy}$ at $1.4 \mathrm{GHz}$ is a limit $\sim 3-5$ times fainter than the constraint from radio fluctuation analysis; it is unlikely that the radio $\log N-\log S$ could sustain its slope below this limit, otherwise the integrated radio emission from weak sources would diverge (Fomalont et al. 1991).

\section{Conclusions}

We have analyzed a small, but well defined sample of 17 starforming galaxies, extracted from the HFS97 catalogue, for which there is a homogeneous information on optical, FIR, radio and X-ray bands (local sample). In agreement with previous work (David et al. 1992) we find that the logarithms of the soft $(0.5-2 \mathrm{keV}) \mathrm{X}$-ray luminosities (corrected for Galactic absorption only) are linearly correlated with the logarithms of both radio $(1.4 \mathrm{GHz})$ and $F I R$ luminosities. We have extended our analysis to the harder X-ray band, essentially free from internal absorption which may affect the soft X-ray fluxes, and found that there is a tight linear correlation between the X-ray luminosities in the $2-10 \mathrm{keV}$ interval with both the radio and the FIR luminosities, normally assumed as the indicators of the star formation rate. The addition of 6 galaxies (supplementary sample) homogeneous with, but not included in HFS97, does not modify these results. We conclude that the origin of the hard X-ray emission must be closely related to star formation and calibrate an X-ray $S F R$ indicator.

Candidate starburst galaxies have been selected in the HDF North, with redshifts up to $z \sim 1.3$, and their rest-frame X-ray luminosities are computed by extracting counts in redshifted bands from the Chandra observation of the HDFN. With this approach we have shown that the $2-10 \mathrm{keV} /$ radio linear correlation holds up to $z \sim 1.3$, encompassing five orders of magnitude in luminosity, up to $L_{2-10} \sim \operatorname{several~} 10^{42} \mathrm{erg} \mathrm{s}^{-1}$ and a corresponding star formation rate $\sim 1000 M_{\odot} \mathrm{yr}^{-1}$. The fit to the $0.5-2.0 \mathrm{keV} /$ radio data is also linear up to $z \sim 1.3$. However, the count ratio for the highest redshift galaxy at $z=1.275$ requires significant absorption if a spectral slope of $\Gamma \sim 2.1$ is assumed; thus this galaxy shows, on a larger scale, the same behaviour as that of the southern core in NGC 3256 (see the appendix). Therefore, while the linearity of the relations involving soft X-ray luminosities remains statistically significant, at high redshift (where galaxies are supposed to have more gas at their disposal to form stars, and so their X-ray emission is more likely to be absorbed) the $2-10 \mathrm{keV}$ luminosity is a more secure indicator of the $S F R$.

As an additional investigation we have also analyzed a sample of LLAGN (LINERs and Seyfert's) included in HFS97 (Terashima et al. 2002): while, as expected, the X-ray luminosities are generally in excess with respect to star-forming galaxies for the same FIR luminosity, the distribution of the objects in the X-ray vs. FIR luminosity diagram is bounded from below from the region occupied by the star-forming galaxies, indicating that the X-ray emission of LLAGN falling in this border-line region could be mainly due to star formation processes, rather than being of nuclear origin.

Our inference of using the $2-10 \mathrm{keV}$ luminosity as a $S F R$ indicator is consistent with a recent study on Lymanbreak galaxies by Nandra et al. (2002) who have extrapolated the David et al. (1992) FIR/soft X-ray relation to the hard X-ray band obtaining a $S F R / 2-10 \mathrm{keV}$ luminosity relation within $10 \%$ of our Eq. (15). From a stacking analysis of Chandra data for a sample of optically selected Lymanbreak and Balmer-break galaxies in the HDFN they find a good agreement of the average $S F R$ as estimated from X-ray and extinction-corrected UV luminosities.

Grimm et al. (2002) have recently claimed that the luminosity function of High Mass X-ray Binaries (HMXB) can be derived from a universal luminosity function whose normalization is proportional to the $S F R$, but only for rates $\gtrsim 1 M_{\odot} / \mathrm{yr}$, while this relation becomes non-linear at lower rates. This appears to be somewhat in constrast with our result that the 2-10 keV luminosity is proportional to $S F R$ even for rates $\$ 1 M_{\odot} / y r$, as supported by the data for 4 galaxies in our sample. However, Grimm et al. 2002 analysis only refers to the contribution from HMXB; the possible contribution of other X-ray components, which are not selected out in our data, might explain this discrepancy.

$\mathrm{X}$-ray number counts for the radio sub-mJy galaxy population are predicted making use of the radio $\log N-\log S$ and of the X-ray/radio correlation. The expected counts extend much below the sensitivities of the deepest X-ray surveys (about one order of magnitude in the soft band, one and a half for the hard 
band), and are within the limits set by the fluctuations analysis in the Chandra deep fields (Miyaji \& Griffiths 2002a, b). They are also consistent with the predictions based on the evolution of the cosmic $S F R$ density by (Ptak et al. 2001). Since the results from fluctuations analysis in the soft X-rays suggest an excess of sources with respect to AGN synthesis models at fluxes below $\sim 10^{-17} \mathrm{erg} \mathrm{s}^{-1} \mathrm{~cm}^{-2}$, it may be possible that the sub-mJy galaxies represent the dominant population in the $\mathrm{X}$ rays at very faint fluxes.

The contribution to the cosmic X-ray background in the $2-10 \mathrm{keV}$ band is estimated by integration of the derived $\mathrm{X}$ ray number counts. The contribution from galaxies detected in the deepest radio surveys is $\$ 2 \%$. This estimate may rise up to $11 \%$ by extrapolating the radio counts down to $1 \mu \mathrm{Jy}$, or $\sim 10^{-18} \mathrm{erg} \mathrm{s}^{-1} \mathrm{~cm}^{-2}$ in the X-ray band. However, since a fraction of the sub-mJy objects may not be star-forming galaxies, these figures for the time being should be regarded as upper limits.

The next step in understanding the physics involved in hard $\mathrm{X}$-ray emission must go through a careful analysis of Chandra and XMM-Newton observations of both low-S FR local galaxies and high-SFR high-redshift ones. Since the explanation of the radio/FIR correlation is still a matter of discussion, we hope that its extension to the X-ray band may help in clarifying this issue.

Acknowledgements. We kindly thank Joe Mazzarella for providing data before publication, and Meri Polletta for very useful comments. We also thank an anonymous referee whose comments have contributed to improve the presentation of this paper. This research has made use of the VizieR database (Ochsenbein et al. 2000) hosted at the Centre de Donnees astronomiques de Strasbourg (CDS), and of data obtained from the High Energy Astrophysics Science Archive Research Center (HEASARC), provided by NASA's Goddard Space Flight Center. This research has been partially supported by ASI contracts $\mathrm{I} / \mathrm{R} / 113 / 01$ and $\mathrm{I} / \mathrm{R} / 073 / 01$, and by the MURST grants Cofin00-02-36 and Cofin-01-02-8773.

\section{Appendix A: Notes on individual galaxies}

\section{IC 342: Variability of ULXs}

In the ASCA observations, the X-ray emission of IC 342 (a face-on spiral galaxy at $3.9 \mathrm{Mpc}$ ) is powered by three main sources; two of them (source 1 and 2 according to Fabbiano \& Trinchieri 1987) are ultraluminous X-ray binaries (ULX) while source 3 is associated with the galactic centre. Observations with higher angular resolution (ROSAT HRI, Bregman et al. 1993) showed that sources 1 and 2 are point-like while source 3 is resolved in at least three sources. Our main concern in determining the flux of this galaxy is the variability of the two ULX, which was assessed by a series of observations spanning several years: IC342 was first observed by Einstein in 1980 (Fabbiano \& Trinchieri 1987), then by ROSAT in 1991 (Bregman et al. 1993), by ASCA in 1993 (Okada et al. 1998) and 2000 (Kubota et al. 2001), and by XMM-Newton in 2001.

In Table A. 1 we report soft X-ray fluxes for sources 1 and 2. We have chosen the soft X-ray band due to the limited energy band of both Einstein and ROSAT; the fluxes observed with
Table A.1. $0.5-2.0 \mathrm{keV}$ fluxes (in $10^{-13} \mathrm{erg} \mathrm{s}^{-1} \mathrm{~cm}^{-2}$ ) for sources 1 and 2 in IC 342 for a blackbody (bb) and a power-law (po) model.

\begin{tabular}{llccc}
\hline \hline & & \multicolumn{2}{c}{ SRC 1 Flux } & SRc 2 Flux \\
YeAR & Mission & BB & PO & PO \\
\hline 1980 & Einstein & 2.7 & 4.1 & 0.85 \\
1991 & ROSAT & 3.3 & 2.7 & 1.3 \\
1993 & ASCA & 16 & & 2.6 \\
2000 & ASCA & & 5.0 & 0.52 \\
2001 & XMM-Newton & & 4.1 & 1.3 \\
\hline
\end{tabular}

these satellites were obtained from the count rates reported in Fabbiano \& Trinchieri (1987) and Bregman et al. (1993) assuming the powerlaw and multicolor disk examined in Kubota et al. (2001) for source 1 and 2 respectively; we take ASCA 1993 and 2000 fluxes from Kubota et al. (2001). XMM-Newton archival observations were reduced by us with SAS 5.3 and the latest calibrations available.

Source 1 was in a low state $\left(F_{0.5-2} \sim 3-5 \times 10^{-13} \mathrm{erg} \mathrm{s}^{-1}\right)$ during the 1980, 1991, 2000 and 2001 observations, and in a high state $\left(F_{0.5-2} \sim 16 \times 10^{-13} \mathrm{erg} \mathrm{s}^{-1}\right)$ during the $1993 \mathrm{ob}-$ servation. The broad-band (0.5-10 keV) spectrum changed, its best-fit model being a disk black-body in 1993 and a power-law in 2000 and 2001. Source 2 has also shown variability, its $0.5-$ $2.0 \mathrm{keV}$ flux oscillating between $0.52 \times 10^{-13}$ (ASCA 2000) and $2.56 \times 10^{-13}$ (ASCA 1993) $\mathrm{erg} \mathrm{s}^{-1}$; the main reason for this variability being the variations in the strongly absorbing column density, which was $9.9 \times 10^{21} \mathrm{~cm}^{-2}$ in 1993 and $18 \times 10^{21} \mathrm{~cm}^{-2}$ in 2000 . The spectrum was always a power-law.

The high state for source 1 seems thus to be of short duration, and we feel confident that its time-averaged flux may be approximated with its low state flux. We thus choose to derive our flux estimate for IC 342 from the ASCA 2000 observation, estimating the variation for the total flux of the galaxy caused by source 2 variability to be less than $10 \%$.

\section{Variability in M 82}

Hard (2-10 keV) X-ray variability in M 82 was reported in two monitoring campaigns with ASCA (in 1996, Ptak \& Griffiths 1999) and RXTE (in 1997, Rephaeli \& Gruber 2002). M82 was found in "high state" (i.e. $4 \times 10^{-11} \lesssim f_{2-10} \lesssim 7 \times 10^{-11}$ ) in three out of nine observations with ASCA and in 4 out of 31 observations with RXTE. In all the other observations it was in a "low state" $\left(2 \times 10^{-11} \lesssim f_{2-10} \lesssim 3.5 \times 10^{-11}\right)$. A low flux level was also measured during the observations with other experiments: HEAO 1 in 1978 (Griffiths et al. 1979); Einstein MPC in 1979 (Watson et al. 1984); EXOSAT in 1983 and 1984; BBXRT in 1990; ASCA in 1993 (Tsuru et al. 1997); BeppoSAX in 1997 (Cappi et al. 1999); Chandra in 1999 and 2000, XMM-Newton in 2001 . No variability was instead detected in the $0.5-2.0 \mathrm{keV}$ band (Ptak \& Griffiths 1999).

The high state has been of short duration: less than 50 days in 1996, when it was observed by ASCA, and less than four months in 1997. A monitoring campaign was also undertaken with Chandra, which observed M 82 four times between September 1999 and May 2000. We reduced the archival data, 


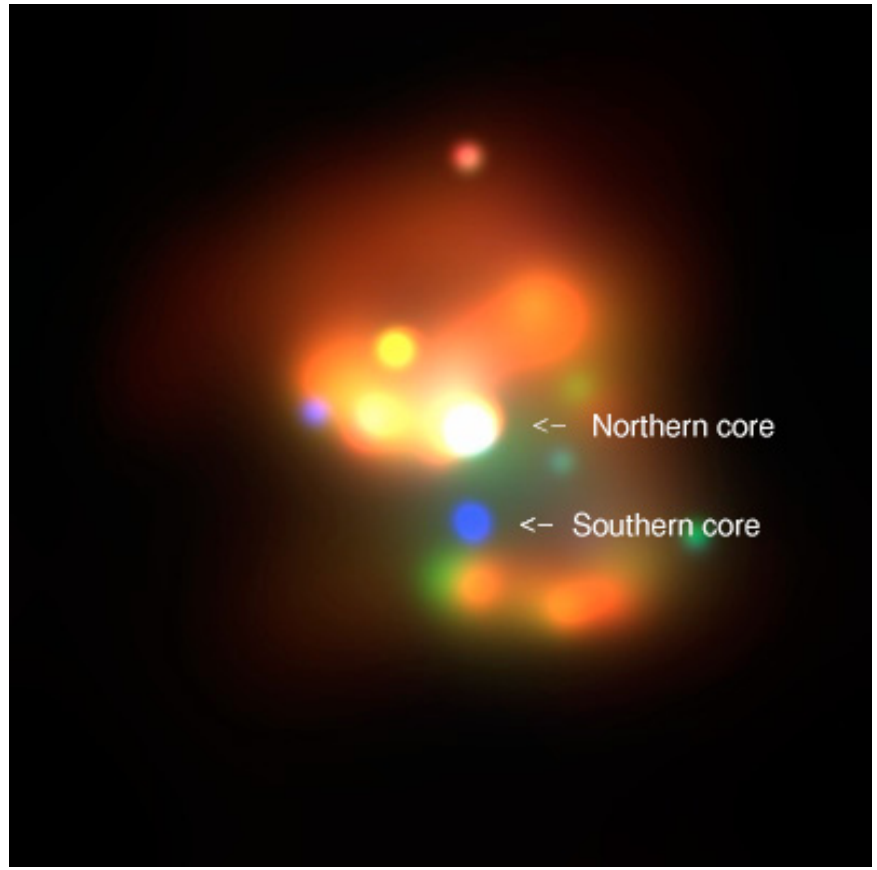

Fig. A.1. True color, smoothed Chandra image of the centre of NGC 3256 (the actual colour is the sum of red, green and blue, the intensity of each one representing the flux in the $0.3-1.0 \mathrm{keV}, 1.0$ $2.0 \mathrm{keV}$ and $2.0-8 \mathrm{keV}$ respectively); north is up, east is left; the distance between the two cores is $\sim 5^{\prime \prime}$. The two cores are the brightest sources both at $1.4 \mathrm{GHz}$ and in the $2-10 \mathrm{keV}$ band, but since the southern one lies behind a dust lane, its soft X-ray emission is completely absorbed. While the northern core follows both the radio/soft X-ray and radio/hard X-ray relations (Eqs. (9), (13)), the southern one only follows the radio/hard X-ray relation.

and found that the galaxy was always in a low state, with its flux slowly increasing from $1.2 \times 10^{-11}$ to $2.9 \times 10^{-11}$.

We do not attempt a detailed analysis of the variability (see Rephaeli \& Gruber 2002); however, we feel confident that, given the short duration of the high states and the fact that the difference between high- and low state flux is about a factor 2, the time-averaged flux of M 82 can be approximated with its low state flux. We thus choose to derive our flux estimate for M 82 from the BeppoSAX 1997 observation $\left(f_{2-10}=2.9 \times 10^{-11} \mathrm{erg} \mathrm{s}^{-1} \mathrm{~cm}^{-2}\right)$, estimating the uncertainty caused by variability to be around $30 \%$.

\section{NGC 3256: A case for intrinsic absorption}

We present the test case of NGC 3256, a luminous dusty merger remnant included in the supplementary sample. Detailed studies at several wavelengths (radio: Norris \& Forbes 1995; IR: Kotilainen et al. 1996; optical: Lípari et al. 2000; X-ray: Moran et al. 1999; Lira et al. 1999) have shown that the energetic output of this galaxy is powered by star formation occurring at several locations, but mainly in the two radio cores discovered by Norris \& Forbes (1995) and also detected with Chandra (Fig. A.1).

The 3 and $6 \mathrm{~cm}$ radio maps (Norris \& Forbes 1995) reveal two distinct, resolved $\left(F W H M \sim 1.2^{\prime \prime}\right)$ nuclei and some fainter diffuse radio emission. Separated by $5^{\prime \prime}$ in declination, the two cores dominate the radio emission, the northern one being slightly (15\%) brighter. They share the same spectral in$\operatorname{dex}(\alpha \sim 0.8)$ and have similar $2-10 \mathrm{keV}$ fluxes. Both of them follow the radio/hard X-ray correlation, while only the northern one follows the radio/soft X-ray correlation. At other wavelengths the northern core is the brightest source in NGC 3256, while the southern one lies behind a dust lane and is only detected in the far infrared $(\lambda>10 \mu \mathrm{m})$, as clearly shown in the sequence of infrared images at increasing wavelengths in Kotilainen et al. (1996).

Although the southern core appears as a bright source in the hard X-rays $(E>2 \mathrm{keV})$, there are not enough counts to allow an accurate spectral fitting. However, it is still possible to constrain the absorbing column density by assuming a template spectrum, such as a simple power-law or the spectrum of the northern core, leading (after standard processing of the Chandra archival observation of NGC 3256) to an intrinsic $N_{\mathrm{H}}=(2.2 \pm 1.0) \times 10^{22} \mathrm{~cm}^{-2}\left(N_{\mathrm{H}, \mathrm{Gal}}=9.5 \times 10^{20} \mathrm{~cm}^{-2}\right)$, fully consistent with the $A_{V}=10.7$ estimated by Kotilainen et al. (1996) from infrared observations. We note that $84 \%$ of the $2-$ $10 \mathrm{keV}$ flux and only $10 \%$ of the $0.5-2 \mathrm{keV}$ one are transmitted through this column density. Thus, while the flux loss in the hard band is still within the correlation scatter, the larger loss in the soft band throws the southern nucleus off the correlation of Eq. (9).

\section{References}

Bookbinder, J., Cowie, L. L., Ostriker, J. P., Krolik, J. H., \& Rees, M. 1980, ApJ, 237, 647

Bauer, F. E., Alexander, D. M., Brandt, W. N., et al. 2002, AJ, 124, 2351

Brandt, W. N., Alexander, D. M., Hornschmeier, A. E., et al. 2001, AJ, 122,2810

Bregman, J. N., Cox, C. V., \& Tomisaka, K. 1993, ApJ, 415, L79

Cappi, M., Persic, M., Bassani, L., et al. 1999, A\&A, 350, 777

Cohen, J., Hogg, D. W., Blandford, R., et al. 2000, ApJ, 538, 29

Comastri, A., Setti, G., Zamorani, G., \& Hasinger, G. 1995, A\&A, 296,1

Condon, J. J. 1992, ARA\&A, 30, 575

Condon, J. J., Helou, G., Sanders, D. B., \& Soifer, B. T. 1990, ApJS, 73,359

Condon, J. J., Helou, G., Sanders, D. B., \& Soifer, B. T. 1996, ApJS, 103,81

Dahlem, M., Weaver, K. A., \& Heckman, T. M. 1998, ApJS, 118, 401

David, L. P., Jones, C., \& Forman, W. 1992, ApJ, 388, 82

Della Ceca, R., Griffiths, R. E., Heckman, T. M., \& MacKenty, J. W. 1996, ApJ, 469, 662

Della Ceca, R., Griffiths, R. E., \& Heckman, T. M. 1997, ApJ, 485, 581

Della Ceca, R., Griffiths, R. E., Heckman, T. M., Lehnert, M. D., \& Weaver, K. A. 1999, ApJ, 514, 772

De Jong, T., Klein, U., Wielebinski, R., \& Wunderlich, E. 1985, A\&A, 147, L6

de Vaucouleurs, G., de Vaucouleurs, A., \& Corwin, H. G. 1976, Second Reference Catalogue of Bright Galaxies (University of Texas Press, Austin) (RC2)

Dobrodiy, O. A., \& Pronik, I. I. 1979, Izv. Astrof. Obs. Krim. (Simeis) LX, 66

Fabbiano, G. 1989, ARA\&A, 27, 87 
Fabbiano, G., \& Trinchieri, G. 1987, ApJ, 315, 46

Fomalont, E. B., Windhorst, R. A., Kristian, J. A., \& Kellerman, K. I. 1991, AJ, 102, 1258

Garrett, M. 2002, A\&A, 384, L19

Garrett, M. A., de Bruyn, A. G., Giroletti, M., Baan, W. A., \& Schilizzi, R. T. 2000, A\&A, 361, L41

Gendreau, K. C., Mushotzky, R., Fabian, A. C., et al. 1995, PASJ, 47, L5

Gilli, R., Salvati, M., \& Hasinger, G. 2001, A\&A, 366, 407

Griffiths, R. E., Schwartz, D. A., Schwarz, J., et al. 1979, ApJ, 230, L21

Griffiths, R. E., \& Padovani, P. 1990, ApJ, 360, 483

Grimm, H. J., Gilfanov, M., \& Sunyaev, R. 2002, MNRAS, submitted [astro-ph/0205371]

Haarsma, D. B., Partridge, R. B., Windhorst, R. A., \& Richards, E. A. 2000, ApJ, 544, 641

Harwit, M., \& Pacini, F. 1975, ApJ, 200, L127

Haynes, R. F., Huchtmeir, W. K. G., \& Siegman, B. C. 1975, Compendium of Radio Measurements of Bright Galaxies, CSIRO Publication

Helou, G., Soifer, B. T., \& Rowan-Robinson, M. 1985, ApJ, 298, L7

Ho, L. C., Filippenko, A. V., \& Sargent, W. L. W. 1995, ApJS, 98, 477

Ho, L. C., Filippenko, A. V., \& Sargent, W. L. W. 1997, ApJS, 112, 315 (HFS97)

Ho, L. C., Feigelson, E. D., Townsley, L. K., et al. 2001, ApJ, 549, L51

Kennicutt, R. C. Jr. 1998, ApJ, 498, 541

Kewley, L. J., Heisler, C. A., Dopita, M. A., \& Lumsden, S. 2001, ApJS, 132, 37

Kotilainen, J. K., Moorwood, A. F. M., Ward, M. J., \& Forbes, D. A. 1996, A\&A, 305, 107

Kubota, A., Mizuno, T., Makishima, K., et al. 2001, ApJ, 547, L119

Lípari, S., Díaz, R., Taniguchi, Y., et al. 2000, AJ, 120, 645

Lira, P., Ward, M., Zezas, A., Alonso-Herrero, A., \& Ueno, S. 2002, MNRAS, 330, 259

Lumb, D. H., Warwick, R. S., Page, M., \& De Luca, A. 2002, A\&A, 389, 93

Mazzarella, J., et al. 2002, in preparation

Miyaji, T., \& Griffiths, R. E. 2002a, ApJ, 564, L5

Miyaji, T., \& Griffiths, R. E. 2002b, Proc. Symp., New Visions of the $\mathrm{X}$-ray Universe in the XMM-Newton and Chandra Era, ESTEC 2001 [astro-ph/0202048]

Moran, E. C., Lehnert, M. D., \& Helfand, D. J. 1999, ApJ, 526, 649

Moshir, M., Copan, G., Conrow, T., et al. 1989, IRAS Faint Source Catalog, $|b|>10$ Degrees, Infrared Processing and Analysis Center
Nandra, K., Mushotzky, R. F., Arnaud, K., et al. 2002, ApJ, 576, 625

Norris, R. P., \& Forbes, D. A. 1995, ApJ, 446, 594

Okada, K., Dotani, T., Makishima, K., Mitsuda, K., \& Mihara, T. 1998, PASJ, 50, 25

Ochsenbein, F., Bauer, P., \& Marcout, J. 2000, A\&AS, 143, 23

Parmar, A. N., Sidoli, L., Oosterbroek, T., et al. 2001, A\&A, 368, 420

Ptak, A., \& Griffiths, R. 1999, ApJ, 517, L85

Ptak, A., Griffiths, R., White, N., \& Ghosh, P. 2001, ApJ, 559, L91

Ranalli, P., Comastri, A., \& Setti, G. 2002, Proc. Symp., New Visions of the X-ray Universe in the XMM-Newton and Chandra Era, ESTEC 2001 [astro-ph/0202241]

Rephaeli, Y., \& Gruber, D. 2002, A\&A, 389, 752

Rice, W., Lonsdale, C. J., Soifer, B. T., et al. 1988, ApJS, 68, 91

Richards, E. A., Kellermann, K. I., Fomalont, E. B., Windhorst, R. A., \& Partridge, R. B. 1998, AJ, 116, 1039

Richards, E. A. 2000, ApJ, 533, 611

Rubin, V. C., Ford, W. K. Jr., \& D’Odorico, S. 1970, ApJ, 160, 801

Rush, B., Malkan, M., \& Spinoglio, L. 1993, ApJS, 89, 1

Sandage, A. R., \& Tammann, G. A. 1981, A Revised ShapleyAmes Catalog of Bright Galaxies (RSA) (Washington: Carnegie Institution of Washington)

Sansom, A. E., Dotani, T., Okada, K., Yamashita, A., \& Fabbiano, G. 1996, MNRAS, 281, 48

Setti, G., \& Woltjer, L. 1989, A\&A, 224, L21

Terashima, Y., Iyomoto, N., Ho, L. C., \& Ptak, A. F. 2002, ApJS, 139,1

Tully, R. B. 1988, Nearby Galaxies Catalog (Cambridge University Press)

Tsuru, T. G., Awaki, H., Koyama, K., \& Ptak, A. F. 1997, PASJ, 49, 619

Ulvestad, J. S., \& Ho, L. C. 2002, ApJ, in press [astro-ph/0208460]

van der Kruit, P. C. 1973, A\&A, 29, 263

Vecchi, A., Molendi, S., Guainazzi, M., Fiore, F., \& Parmar, A. N. 1999, A\&A, 349, L73

Veilleux, S., \& Osterbrock, D. E. 1987, ApJS, 63, 295

Watson, M. G., Stanger, V., \& Griffiths, R. E. 1984, ApJ, 286, 144

Webster, B. L., \& Smith, M. G. 1983, MNRAS, 204, 743

Windhorst, R. A., Miley, G. K., Owen, F. N., Kron, R. G., \& Koo, D. C. 1985, ApJ, 289, 494

Windhorst, R. A., Mathis, D. F., \& Neuschaefer, L. W. 1990, Proc. Symp., Evolution of the universe of galaxies: Edwin Hubble Centennial Symposium, ASP Conf. Ser., 10, 389

Yun, M. S., Reddy, N. A., \& Condon, J. J. 2001, ApJ, 554, 803

Zezas, A. L., Georgantopoulos, I., \& Ward, M. J. 1998, MNRAS, 301, 915 